\title{
Thematische Kartierungen mit Hilfe von multidimensionalen Satellitendaten
}

\section{EINLEITUNG}

Thematische Kartierungen, z.B. der Landnutzung, veralten schnell. Nachführungen werden, wenn überhaupt, in viel zu langen Zeitabständen vorgenommen. Rasch veränderliche Landschaftselemente (Schnee/Vegetationswachstum etc.), der Ablauf dynamischer Landschaftsprozesse, lassen sich im grossräumig vergleichenden Ueberblick überhaupt nicht am Boden erfassen. Die technische Entwicklung spezifischer Erderkundungssatelliten eröffnet der Geographie ganz neue Möglichkeiten zur schnellen, routinemässigen Neuerstellung und Nachführung kleinmassstäbiger thematischer Karten.

Voraussetzung für diese neuartige Form der Informationsbeschaffung sind multidimensionale Datensätze (Kap. 3). Diese müssen uns systematisch über längere Zeit hinweg durch ein geeignetes Satellitensystem übermittelt werden, das für praktische Anwendungen folgenden Anforderungen genügen muss: Kontinuität in der Aufnahme, kurze Wiederholungsrate, hohe Bodenauflösung. Diesen Ansprüchen vermag heute einzig das amerikanische LANDSAT-System (früher ERTS) einigermassen zu genügen. Die operationelle Erfassung raschändernder Phänomene an der Erdoberfläche als Grundlage für Inventuren, Ernteprognosen, Abflussvorhersagen etc. bedingt zudem eine sehr schnelle Zustellung der Daten an den Benützer. Mit dem von der europ. Weltraumbehörde ESA geschaffenen Datenempfangs-, Verarbeitungs- und Verteilungsnetz EARTHNET wurden in Europa die entsprechenden Voraussetzungen für einen kontinuierlichen Datenempfang und eine rasche Verfügbarkeit geschaffen.

Das LANDSAT-System wurde an anderer Stelle schon mehrfach eingehend beschrieben $(1,2,3)$. Das EARTHNET soll dagegen im folgenden kurz vorgestellt werden. Dazu werden Bedeutung und Probleme der Interpretation multidimensionaler Datensätze für thematische Kartierungen diskutiert und anhand von Beispielen aus dem europ. Bereich illustriert.

\section{EARTHNET-PROGRA:1:-1}

Es bezweckt die Errichtung eines Datennetzes für Erderkundungssatelliten, das den kontinuierlichen Empfang, die rasche Aufbereitung (Umwandlung der Daten in Standard-Ausgabeprodukte), Verteilung und die Archivierung der Daten zu erschwinglichen Konditionen gewährleistet.

Für den direkten Datenempfang von LANDSAT sind in Europa zwei Bodenstationen notwendig (Fig.l).

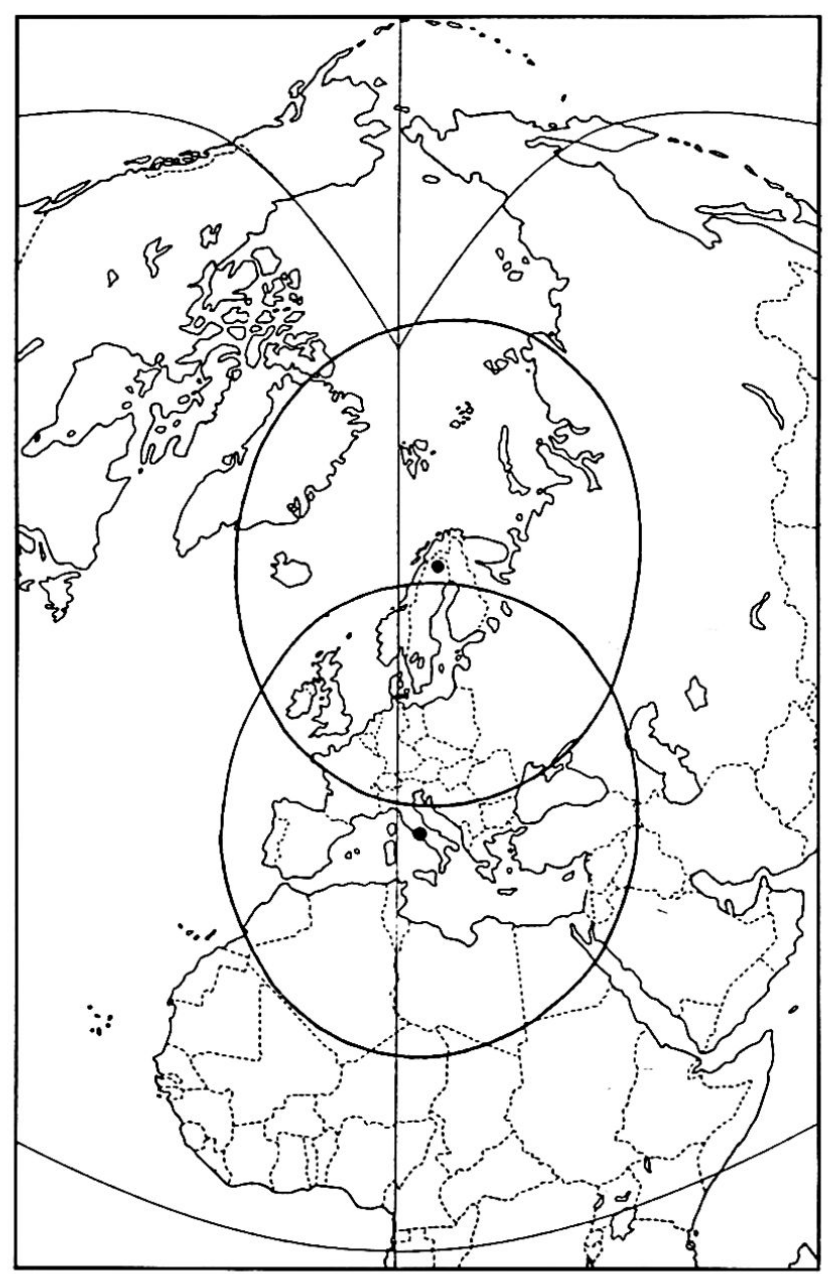

Fig. 1 : Empfangsbereich der Bodenstationen Kiruna (Schweden) und Fucino (Italien) für LANDSAT-Daten.

Die südliche Station steht in Fucino, ca.120 km östlich von Rom. Sie ist seit Früjahr 1976 operationell. Die nördliche Bodenstation wird in Kiruna eingerichtet; sie soll ihren Betrieb im Frühling 1978 aufnehmen. Für 1978 sind weitere Fernerkundungssatelliten experimenteller Art (SEASAT-A, HCMM, NIMBUS-G) vorgesehen (3).

Prof. Dr. Harold Haefner, Geographisches Institut Universität Zürich, Blüml isalpstrasse 10 8006 Zürich 
Entsprechende Empfangsstationen und Datenverarbeitungsstellen sind in Vorbereitung und sollen sukzessive in das Datennetz integriert werden.

Das zentrale Datenverteilungs- und Informationszentrum entstand am ESRIN in Frascati. Dem bereits existierenden computerisierten Dokumentationssystem (RECON-System) mit Terminals in allen Mitgliedstaaten wird ein Uebersichtskatalog angegliedert, der Informationen über Lage, Qualität, Bewölkungsgrad etc. jeder einzelnen Aufnahme enthält oder für ein gewünschtes Gebiet Angaben über die vorhandenen Aufnahmen macht. Der Benützer erhält so direkten Zugriff zu den registrierten Aufnahmen und kann umgehend seine Bestellung plazieren (4)..0ffen bleibt die Frage eines zentralen Archivs. Man muss sich bewusst sein, dass die anfallenden Datenmengen enorm sind. Allein zwischen April 1975 und November 1976 hat Fucino von LANDSAT - 1 rund 35'000 Aufnahmen (à 4 Einzelbilder) mit weniger als 50\% Bewölkungsanteil aufgenommen.

\section{INTERPRETATION MULTIDIMENSIONALER DATEN - SAETZE}

Mit den herkömmlichen Verfahren ist eine fristgerechte und umfassende Interpretation der genannten grossen Datenmengen ausgeschlossen. Es gilt, neue (computerunterstuitzte) Methoden zu entwickeln, die im gleichen Tempo, in dem die Daten empfangen werden, daraus die gewünschte wissenschaftliche Information herzuleiten vermögen. Benötigt werden also automatisierte Klassifikationssysteme zur Interpretation von multidimensionalen Datensätzen. Darunter sollen Datensätze verstanden werden, von denen in zwei oder mehreren Dimensionen Mehrfach-Messwerte vorliegen. Es werden vor allem unterschieden :

Multispektral-Daten: Messung der reflektierten resp. emittierten Strahlung in mehreren Spektralbereichen ( bei LANDSAT in 4 Bandbereichen)

Multitemporal-Daten: Messung desselben Objekts $z u$ verschiedenen Aufnahmezeitpunkten (bei LANDSAT alle 18 Tage)

Multispatial - Daten: Messung der Objekte in ihren mehrdimensionalen Beziehungen zur Umgebung; simultane Verwendung von Aufnahmesystemen mit unterschiedlicher Bodenauflösung (LANDSAT ca. $80 \times 80 \mathrm{~m})$.

Multispektraldaten erlauben die Benützung des gesamten optischen Wellenlängenbereichs des elektromagnetischen Spektrums. Durch die Kombination geeigneter Spektralbänder resultiert eine Steigerung der interpretierbaren Information und der Klassifikationsgenauigkeit.

Es bestehen bereits zahlreiche Methoden der automatisierten Datenverarbeitung; wir verweisen auf entsprechende Literatur $(2,3,5,6)$. Festgehalten sei, dass jeweils die folgenden drei Teilschritte auseinandergehalten werden sollen:
- Datenvorverarbeitung

- Datenklassifikation

- Informationsausgabe (Darstellung der Ergebnisse)

Das methodische Vorgehen hat sich dabei auf die verfügbaren Geräte auszurichten. Wichtig ist, dass die Teilschritte sorgfältig aufeinander abgestimmt sind. Besonderes Augenmerk ist dabei auf eine adaequate Informationsausgabe zu richten um z.B. die Vorlagen für thematische Karten herstellen zu können (7).

Der Hauptvorteil von Satellitendaten liegt aber in der Möglichkeit der Verwendung multitemporaler Datensätze. Dies in zweifacher Hinsicht. Das Einbeziehen von Aufnahmen von verschiedenen Jahreszeiten ergibt zusätzliche Parameter im Klassifikationsverfahren und gestattet damit eine eindeutigere Identifizierung und Abgrenzung bestimmter Einheiten. Ferner lassen sich aus dem Vergleich mehrerer Aufnahmedaten Landschaftsveränderungen kartieren und durch Kombination von Einzelsituationen in systematischen Zeitabständen ein detaillierteres Bild über dynamische Landschaftsprozesse gewinnen. Wenn einmal die Methoden entwickelt und automatisiert sind, so kann die Datenverarbeitung operationell und mit verhältnismässig geringem Aufwand laufend durchgeführt werden. Zeitgerechte Nachführungen, Landnutzungskartierungen grösserer Gebiete, die auch jahreszeitliche Aspekte mitbeinhalten, sind damit in den Bereich der Realität gerückt.

Die direkte Verwendung von multitemporalen Datensätzen in computerisierten Auswertesystemen bedarf allerdings weitreichender Vorverarbeitungsschritte zur Korrektur und Standardisierung der Daten. Sie berühren sowohl die geometrischen als auch die radiometrischen Aspekte.

Die geometrischen Probleme ergeben sich aus der Tatsache, dass jedes einzelne Bildelement ( $\mathrm{Pi}$ xel) einer Aufnahme genau bezüglich seiner Lage lokalisiert werden muss, damit es mit dem entsprechenden Bildelement einer zeitlich nachfolgenden Aufnahme verglichen werden kann. Da sich die Bahnparameter des Satelliten (Aufnahmemittelpunkt, Tilt etc.) aber von einem Ueberflug zum anderen leicht verschieben, ist dies eine komplizierte Angelegenheit. Entsprechende Untersuchungen sind im Gange, um die notwendigen Voraussetzungen für eine routinemässige Datenverarbeitung sicherzustellen $(8,9)$.

Die spektralen Werte, die der Satellit registriert, werden durch atmosphärische Einflüsse modifiziert. Da sich der Zustand der Atmosphäre von einem Ueberflug zum anderen erheblich verändern kann, müssen diese Effekte erfasst und die Daten entsprechend korrigiert werden können, d.h. die Messdaten sind für einen Vergleich zu standardisieren. Wesentliche Faktoren, die bestimmt werden müssen, sind die atmosphärische Transmission und der Streulichtanteil. Zusätzliche Schwierigkeiten ergeben sich im alpinen Gelände aus der kleinräumigen regionalen Differenzierung im Zustand der Atmosphäre und aus den 
grossen Höhenunterschieden. Auch diese Probleme werden gegenwärtig untersucht (10).

Zusammenfassend sei festgehalten, dass mittels einer geeigneten Datenorganisation und der Einführung von geometrischen Korrekturen im Vorverarbeitungsschritt, sowie dem Einsatz eines adaequaten Informationsausgabesystems eine bildhafte Darstellung der Klassifikationsergebnisse in kartengerechter Form, d.h. einem gewünschten Massstab erreicht werden kann.

\section{KARTIERUNGSBEISPIELE}

Anwendungsgebiete für thematische Kartierungen mittels Satellitendaten im europäischen Bereich sind insbesondere:

- Land- u. Forstwirtschaft:

Anbauflächen, Rotationsmuster, Wirtschaftspläne, Ertragsschätzungen etc.

- Hydrologie:

Schneedecke, Abflussvorhersage, Bodenfeuchtigkeit, Niederschlagsgebiete etc.

- Siedlungsplanung:

Städtische Landnutzung, Wachstumsmuster etc.

- Küstengebiete:

Eisbildung, Verschmutzungen, Sedimentation etc

Das Ergebnis einer Satellitendaten-Klassifikation kann demnach in kartengerechten Vorlagen bestehen, die die räumliche Verbreitung einzelner thematischer Einheiten lagerichtig wiedergeben. Durch kartographische Weiterverarbeitung (Generalisierung, Kombination mit topographischer Unterlage etc.) entstehen druckfertige Themenkarten. Die wiederholte Aufnahme und Kartenherstellung erlaubt das Festhalten von Landschaftsveränderungen. Neben den lagemässigen Veränderungen lassen sich auch die entsprechenden Flächenanteile berechnen. Je ein Beispiel zur Landnutzungsund Schneekartierung soll diese Möglichkeiten illustrieren.

\subsection{Landnutzungskartierung in der Poebene}

Mit Hilfe eines digitalen Datensatzes (7.0ktober 1972) konnte im Raume Mailand eine Landnutzungskartierung mit den folgenden Kategorien erstellt werden (2):

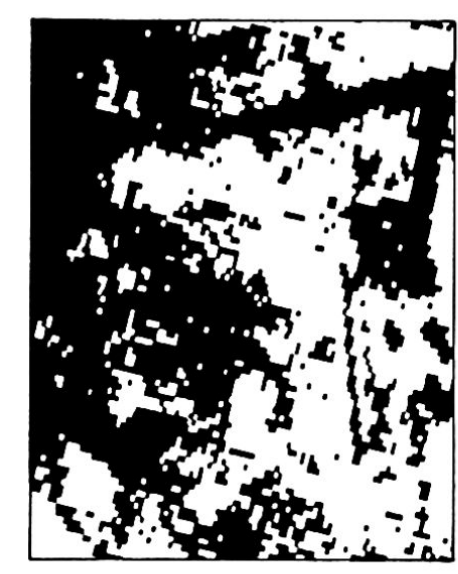

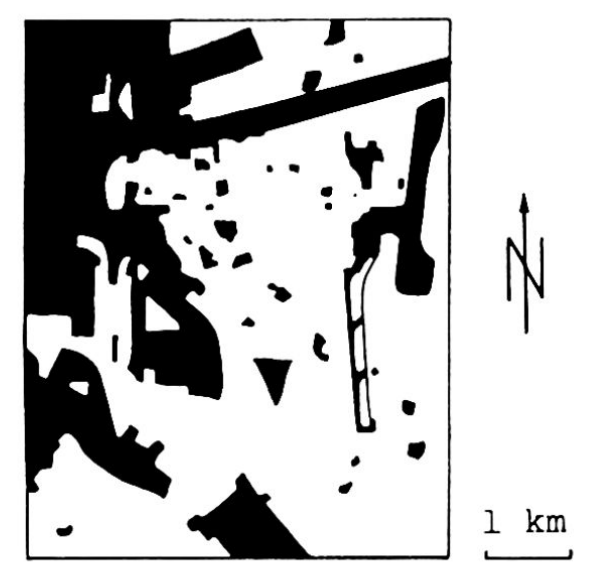

- Wasser

- Ueberbaute Flächen, unterteilt in Grossiedlungen (dichte Bebauung), Kleinsiedlungen (lockere Bebauung), grosse Verkehrs- u. Industriekomplexe (Flughafen, Grossfabriken etc.)

- Landwirtschaftliche Flächen mit grüner Vegetation (spez. Wässerwiesen)

- Landwirtschaftliche Flächen mit toter Vegetation (abgeerntete Felder)

- Wald.

Gesamthaft wurde ein Gebiet von $1650 \mathrm{~km}^{2}$ (fast die Fläche des Kt.Zürich), was 362 '192 Bildelementen entspricht, bearbeitet. Die Informationsausgabe erfolgte mit dem Photomationsystem in Schwarzweiss-Darstellung für jede Kategorie gesondert im Massstab 1:200'000. Die Vorlagen wurden anschliessend eingefärbt, übereinandergepasst und so ein Farbkomposit erstellt. Eindrücklich kommt darin die radiale Entwicklung von Mailand entlang den Ausfallachsen und die Fontanilizone zwischen der Alta Pianura und der Bassa Pianura mit den noch grünen Marciten zum Ausdruck. Fig. 2 zeigt einen Vergleich zwischen Satellitendatenklassierung und konventioneller Landnutzungskarte. Auch unter Berücksichtigung der unterschiedlichen Aufnahmedaten (ca. 3 Jahre) bestechen die Uebereinstimmung und der wesentlich höhere Detaillierungsgrad der Satellitendatenauswertung.

\subsection{Schneedecke in der Landschaft Davos}

Das in Fig. 3 dargestellte Kartierungsbeispiel (11) veranschaulicht eine ganze Reihe der besprochenen Probleme:

- Bei der Klassifikation der Schneedecke wurde insbesondere auf eine sorgfältige Festlegung der Schneegrenze, d.h.der Uebergangszone zwischen total schneebedeckt und völlig aper, Wert gelegt (Fig.3b).

- Auch bereits relativ kleine Testgebiete (hinteres Dischmatal, ca.40 $\mathrm{km}^{2}$ ) lassen sich mit Satellitendaten bereits genau kartieren. Die erzielten Resultate sind mindestens so genau wie diejenigen von konventionellen Luftaufnahmen (11).

- Die Ausdehnung der Schneedecke sollte hier als wichtigster Faktor für ein Abflussmodell (12) dienen. Deshalb musste nicht einfach ein beliebig abgrenzbarer rechteckiger Ausschnitt, son-

Fig. 2:

Vergleich der LANDSAT-Satellitenauswertung (links) mit konventioneller Landnutzungskartierung (rechts) Stadtrand von Mailand im SE mit Flughafen Linate (aus BINZEGGER, 2 ) schwarz: alle nicht vegetationsbedeckten Flächen. 


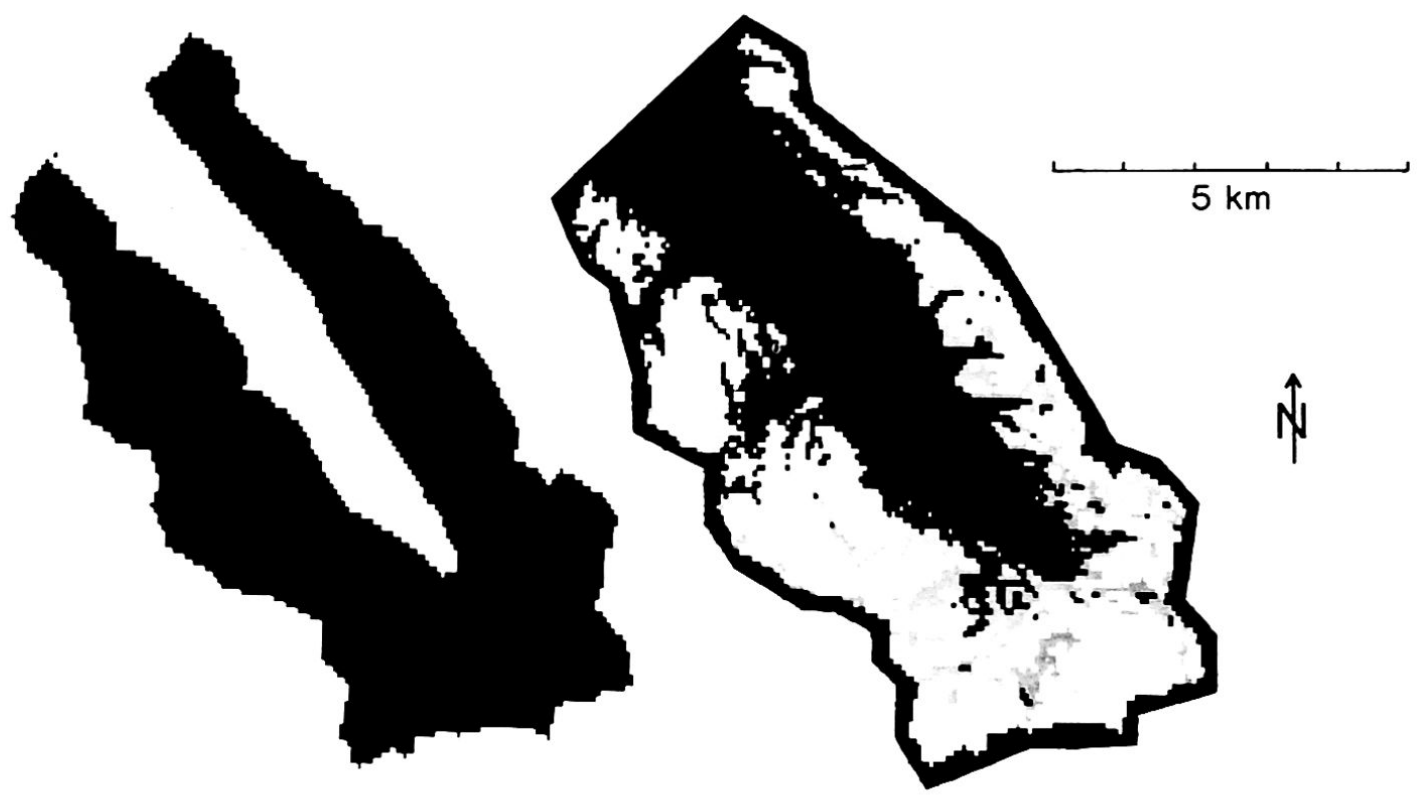

Fig.3: Automatisierte Schneedecken-Klassifikation u. Berechnung der Flächenanteile in verschiedenen Höhenstufen - Hinteres Dischmatal, Davos (aus URFER, 11).

$$
\begin{aligned}
& \text { links: Höhenstufen } \\
& \text { hellgrau : } 1600-2100 \mathrm{~m} \\
& \text { dunkelgrau: } 2100-2600 \mathrm{~m} \\
& \text { schwarz : 2600-3100 } \mathrm{m}
\end{aligned}
$$

$$
\begin{aligned}
& \text { rechts: Schneebedeckung am } 8.6 .76 \\
& \text { weiss : völlig schneebedeckt } \\
& \text { grau : Vebergangszone } \\
& \text { schwarz: völlig schneefrei }
\end{aligned}
$$

dern ein unregelmässig begrenztes Einzugsgebiet, unterteilt in einzelne Höhenstufen, klassiert werden. Um das zu erreichen, wurde ein zusätzlicher Datensatz der Geländeoberfläche in gleich grossen räumlichen Einheiten wie die LANDSATBildelemente digitalisiert (Fig.3a). Anhand von eindeutig lokalisierbaren Geländepunkten kann nun dieser Gelände-Datensatz jeweils mit dem Satelliten-Datensatz zur Deckung gebracht und die Schneedeckenanteile für jede Höhenstufe getrennt berechnet werden.

Damit können die für die Abflussvorhersage wichtigsten Informationen schnell und zuverlässig hergeleitet werden.

\section{AUSBLICK}

Die Entwicklung der notwendigen Software zur Klassierung multidimensionaler Satellitendaten als Informationsquelle für thematische Kartierungen schnelländerlicher Landschaftselemente ist sehr aufwendig, da zahlreiche systemsbedingte und atmosphärische Einzelprobleme mitberücksichtigt und den europäischen Verhältnissen entsprechend gelöst werden müssen. Nachher aber wird ein routinemässiger, kontinuierlicher Informationsfluss mit relativ. geringem Aufwand möglich sein, der aktuellste und mit andern Methoden nicht erreichbare wissenschaftliche Ergebnisse erbringt. Im Vordergrund stehen heute die Abgrenzung flächenhafter Phänomene. Darüber hinaus geht die Forschung aber bereits weiter in Richtung von quantitativen Erhebungen. Neben der flächenhaften Bestimmung spielen Zustandserfassungen etc. Auf diesem Weg iassen sich aktueilste Unterlagen gewinnen zur Ueberwachung und Produktionslenkung unserer wichtigsten naturlichen Ressourcen.
Anmerkungen

1)NASA: LANDSAT Data Users Handbook; NASA Goddard Space Flight Center, Greenbelt 1976.

2)BINZEGGER R.P.: ERTS Multispektraldaten als Informationsquelle für themat. Kartierungen; Diss. Univ. Zürich 1975.

3) HAEFNER H.: Erderkundungssatelliten; NZZ, Forschung u. Technik, 1.2. 1978.

4)Bestellungen sind zu richten an: EARTHNET Business Officer, ESRIN, Via Galileo Galilei, Casella Postale 64, I-0044 Frascati.

5)SEIDEL K.: Digitale Datenerfassung; Techn.Bericht, Photogr. Institut ETH Zürich, 1976.

6)MAXWELL E.L.: Multivariate System Analys is of Multispectral Imagery;Photogrammetric Engineering and Remote Sensing, XLII/No 9,Sept.1976.

7) Am Photograph. Institut der ETHZ steht das Bildverarbeitungssystem PHOTOMATION P $1700 \mathrm{der}$ Firma OPTRONICS Int. zur Verfügung.

8)LICHTENEGGER J. ,SEIDEL K. ,KUEBLER 0.: Methoden zur Ueberlagerung von LANDSAT-Bildern für multitemporale Landnutzungskartierung; erscheint in Bildmessung $u$. Luftbildwesen 1978.

9) LICHTENEGGER J.: Multivariable Datenanalyse von LANDSAT-Aufnahmen an einem Testgebiet mit versch. Landnutzung im Grossen Moos (Prov. Arbeitstitel, Diss. Univ.Zürich, erscheint 1978).

10)STAENZ K.: Atmosphärische Korrekturen von multitemporalen LANDSAT-Daten (Prov.Arbeitstitel, Diss.Univ.Zürich,erscheint 1978).

11)URFER H.P.: Automatisierte Schneekartierung in versch.Flusseinzugsgebieten (Prov. Arbeitstitel, Dipl.Arbeit, Univ.Zürich,erscheint 1978).

12)MARTINEC J.: Hydrologic Basin Models; ISPRACourses, Remote Sensing Applications in Hydrology; Joint Research Centre, Ispra 1977. 